\title{
Correction to: Acetylcholinesterase inhibitors and the risk of osteoporotic fractures: nested case-control study
}

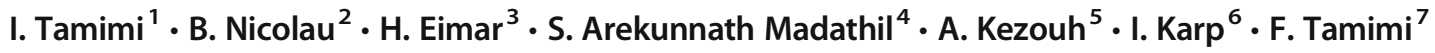

Published online: 15 April 2019

(C) International Osteoporosis Foundation and National Osteoporosis Foundation 2019

\section{Correction to: Osteoporosis International (2017) https://doi.org/10.1007/s00198-017-4346-z}

Please find below the correction for the paragraph under the Heading "Materials and methods - Regulatory approval":

The study was approved by the Scientific and Ethical Advisory Group from the Clinical Practice Research
Database (CPRD; reference protocol 17_172 25) (previously known as the General Practice Research Database) and the ethics review board of the McGill University Health Centre.

Publisher's note Springer Nature remains neutral with regard to jurisdictional claims in published maps and institutional affiliations.

The online version of the original article can be found at https://doi.org/ 10.1007/s00198-017-4346-z

F. Tamimi

faleh.tamimimarino@mcgill.ca

I. Tamimi

isktamimi80@yahoo.com

1 Hospital Regional Universitario de Malaga, Avenida Carlos Haya SN, 29010 Malaga, Spain

2 Division of Oral Health and Society Research, Faculty of Dentistry, McGill University, 2001 McGill College Avenue, Montreal, QC H3A 1G1, Canada

3 Faculty of Medicine and Dentistry, University of Alberta, 2J2.00WC Mackenzie Health Sciences Centre 8440112 St. NW,

Edmonton, Alberta T6G 2R7, Canada
4 Division of Oral Health and Society Research, Faculty of Dentistry, McGill University, 2001 McGill College Avenue,

Montreal, QC H3A 1G1, Canada

5 Department of Epidemiology and Biostatistics, Centre for Clinical Epidemiology, Lady Davis Institute, 3755 Cote-Ste-Catherine Road, Montreal, QC H3T 1E2, Canada

Department of Epidemiology and Biostatistics, Kresge Building K214, Western University, London, Ontario N6A 5C1, Canada

\footnotetext{
Faculty of Dentistry, McGill University, 3640 University Street,
} Montreal, QC H3A 2B2, Canada 